\title{
Different Models for the Simulation of DC Railway Systems supplied by Non-Reversible Substations
}

\author{
C. Mayet ${ }^{1}$, P. Arboleya ${ }^{2}$, A. Bouscayrol ${ }^{3}$, B. Mohamed ${ }^{2}$, P. Delarue ${ }^{3}$, and I. El-Sayed ${ }^{2}$ \\ 1. SATIE - UMR CNRS 8020, Conservatoire National des Arts et Métiers, HESAM University, F-75003 Paris, France \\ 2. LEMUR Research Group, University of Oviedo, Gijon Campus, Asturias 33204 Gijon, Spain \\ 3. Univ. Lille1, Centrale Lille, Arts et Métiers Paris Tech, HEI, EA 2697 - L2EP - Laboratoire d'Electrotechnique et \\ d'Electronique de Puissance, F-59000 Lille, France \\ Contact author: clement.mayet@lecnam.net
}

\begin{abstract}
Simulation is a valuable way to develop efficient design of the infrastructure and operations of electrical railway systems. Nevertheless, the size of such systems and the complexity of the power flows lead to complex models that require simplifications. A specific scenario has been identified as particularly difficult to study with most of the existing simulation tools. This scenario consists in a line with low energy recovery capacity in which all the substations are blocked. In such case, the railway system is disconnected from the AC grid and becomes an islanded system in which the braking trains, and eventually energy storage subsystems, supply other trains in traction mode. This paper aims to compare different modelling approaches to simulate DC railway systems in this specific scenario. The results highlight that conventionnal approach is not able to deal with this scenario. Another approach, based on a dynamic model from previous research works, is well able to estimate the system behavior with this scenario, but it leads to long computational time. Finally, an intermediary approach is proposed to simplify the model in such scenario and potentially reduce the computation time.
\end{abstract}

Keywords-DC Traction System, Non-reversible Substation, Dynamic and Quasi-Static Models, Energetic Macroscopic Representation (EMR), Modified Nodal Analysis.

\section{INTRODUCTION}

Nowadays, transportation systems are key players to face current challenges in terms of energy saving and greenhouse gas emissions [1]. Public transportation systems, such as electric railways, are currently employed in urban areas due to their high transportation capacities and because they do not produce local pollutant emission [2]. However, the global warming and increasing cost of energy lead to still develop more efficient systems. Innovative solutions have been introduced to save more braking energy, such as on-board or wayside Energy Storage Systems (ESS) [3]-[5] and reversible Traction Power Substations (TPS) [6], [7]. However, these solutions induce supplementary cost and their effectiveness must be assessed before their real implementations.

Several simulation tools have been proposed in the last decade to study railway systems and to estimate the potential energy that can be saved [8]-[11]. Conventional TPS (nonreversible), that connect the AC grid to the DC traction network, and the squeeze control (over-voltage protection) play an important role in the solving procedure of railway simulation tools. Indeed, the conventional TPS induces non-linear behavior when it switches between on- and off-states [12]. The squeeze control of a braking train is activated when the supplied DC voltage is too high. In such case, the braking energy is dissipated in the rheostatic brake instead to be recovered on the DC network and induces energy discontinuities from the DC network point of view. Several simplifications on the models are commonly accepted due to the complexity of such systems.

A specific scenario has been identified as particularly difficult to study with most of the existing simulation tools due to the widely accepted simplifications. This scenario consists of a low receptive line with most of the trains in braking mode. All the TPSs are thus blocked, which disconnect the railway system from the AC grid. It becomes an islanded system in which the braking trains supply the other consumers. In such case, some simulation tools neglect what happens in the system during this period because no energy is provided by the AC grid [5], [9]. Other simulation tools use complex iterative methods to approach the mathematical solution [8], [11]. The inability of most of the simulation tools to deal with the case where all the TPSs are blocked can conduct to approximations in the design of potential innovative subsystems. Indeed, their sizing is sensitive because they are based on a cost-benefit calculation based on potential energy savings. However, these innovative subsystems are especially used when the line receptivity is low to save the braking energy, which would be dissipated in the conventional railway system. Furthermore, the control of ESS or reversible TPS is generally based on the DC network voltage [4], [5], [6], [11]. It is therefore crucial to be able to accurately estimate this voltage.

The objective of the paper is to compare different modelling methodologies to simulate DC railway systems supplied by non-reversible TPSs. The periods when the line receptivity is low, which conduct to block all the TPSs, are especially studied. The paper is then organized as follows. The studied case is presented in section II. Section III describes the models of the different subsystems of railway systems. Section IV is focused on different modelling approaches to simulate the entire systems. The simulation results are then compared and analyzed in Section V. 


\section{DESCRIPTION OF THE STUDIED CASE}

A specific scenario has been defined in order to analyze the simulation results and compare the different simulation approaches. A conventional light rail DC system with a $6.8 \mathrm{~km}$ line composed of 7 passenger stations (PS) and 3 conventional TPSs is considered (Fig. 1 and Fig. 2). The 3 TPSs are supposed identical and have been previously defined in [12]. The vehicles are light automatic subways, which have been described in [13]. The studied scenario considers the headway of 120s, which requires 10 trains in circulation for a simulation time of $1000 \mathrm{~s}$. Furthermore, it is assumed that there is no traffic initially on the line. The different trains (T1 to T10) are thus injected on the line from PS1 and PS7 (Fig. 3). The velocity profile has been defined based on the respect of the kinematical limitations (torque, speed, acceleration, voltage, etc.).

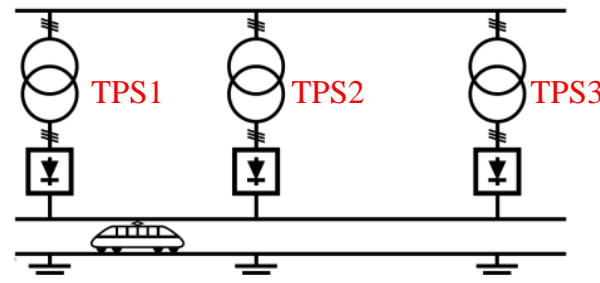

Fig. 1. Electrical description of the railway system (3 TPSs).

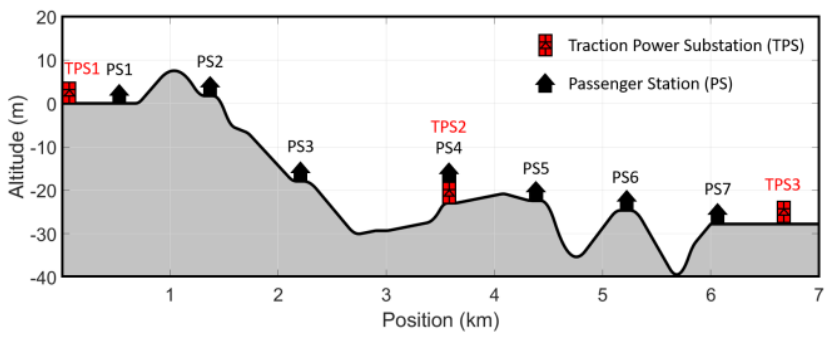

Fig. 2. Profile description of the studied line.

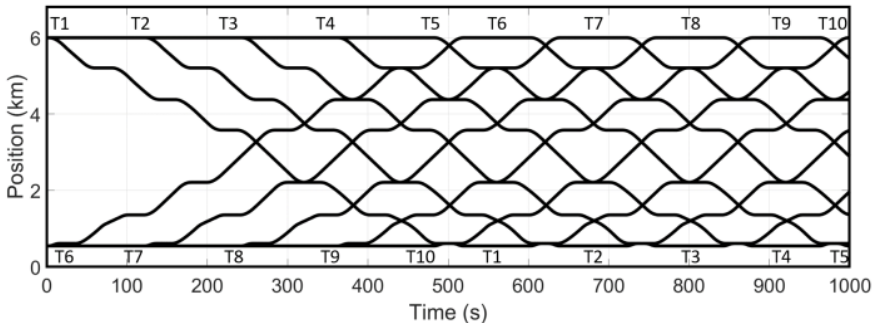

Fig. 3. Schedule of the different trains.

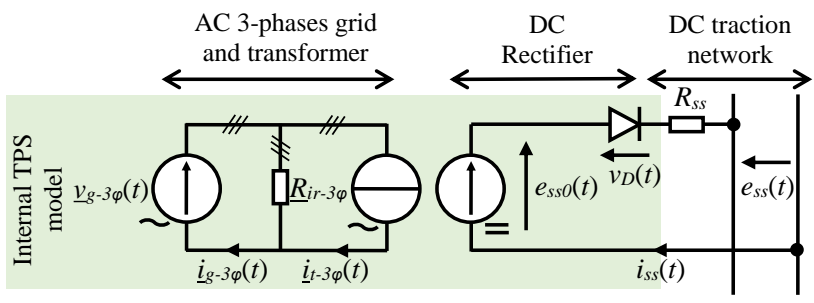

Fig. 4. Equivalent electric circuit of the TPS.

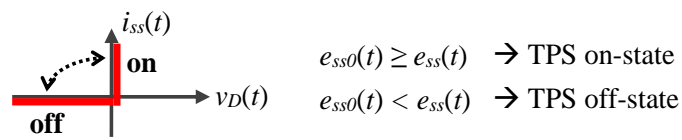

Fig. 5. TPS model switching conditions with perfect diode characteristics.

\section{MODELS OF THE BASIC RAILWAY SUBSYSTEMS}

This section describes the models of the basic subsystems of railway systems (i.e. TPS, Train, DC network). These models are organized using the Energetic Macroscopic Representation (EMR). The EMR is a graphical description tool, which highlights the energy properties of a system [14]. It organizes the system into interconnected basic elements. All elements are connected according to the interaction principle: the product of the action and reaction variables leads to the power exchanged by connected elements. Furthermore, all the components are described respecting the physical integral causality.

\section{A. Model of the Conventional Traction Power Substations}

Neglecting harmonics, the equivalent circuit (Fig. 4) of a conventional TPS is described using a switched-model to estimate its on- and off-states behaviors (Fig. 5 and Fig. 6) [12]. The on-state model imposes to the DC network the rectified open-circuit voltage $e_{s s}(t)$ through the resistance $R_{s s}$ (TPS Joule losses). It estimates the 3 -phases AC currents $\underline{i}_{g-3 \varphi}(t)$ supplied by the AC grid according to the DC current $i_{s s}(t)$ and the resistance $R_{i r-3 \varphi}$ (transformer iron losses). The off-state model imposes currents equal to zero on the TPS DC $\left(i_{s s}(t)=0\right)$ as well as AC $\left(\underline{i}_{t-3 \varphi}(t)=\underline{0}\right)$ sides. Switching conditions are defined (Fig. 5). Note that some models increase the value of the resistance $R_{s S}$ in off-state instead of using a current-voltage switching model [5], [8]. With such an approach, the choice of the value of $R_{s s}$ in offstate can impact the computation time or the simulation results themselves. More explanations about this TPS model are in [12].

\section{B. Model of the Trains}

The train model considers the different components of the train [13]. It determines the electric power $P_{\text {ref }}(t)$ absorbed or recovered on the train input filter by the traction and auxiliary subsystems. The auxiliary power $P_{a u x}(t)$ is generally assumed known. The traction power $P_{\text {trac }}(t)$ is estimated according to the line profiles (slope, speed, etc.), the mass of the vehicle, the resistive efforts (friction, aerodynamic, etc.), the components losses, and the electro-kinematical limitations (torques/currents, acceleration, speed, voltage, etc.). More details can be found in [13]. The train equivalent circuit (Fig. 7) consists in a current source $i_{\text {ref }}(t)$, which represents the traction and auxiliary subsystems, connected in parallel with the rheostatic brake and the DC bus capacitor $C_{f}$. The brake is activated according to the squeeze control (overvoltage protection), which induces a braking current $i_{R}(t)$ depending on $i_{\text {ref }}(t)$ and $u_{f}(t)$ (Fig. 8). All the braking energy is therefore dissipated in the brake when the maximal voltage $U_{2}$ is reached. The braking coefficient $k_{R}(t)$ is increased linearly from $U_{1}$ to $U_{2}$. Finally, the resistance $R_{f}$ represents the losses in the filter as described in [13].

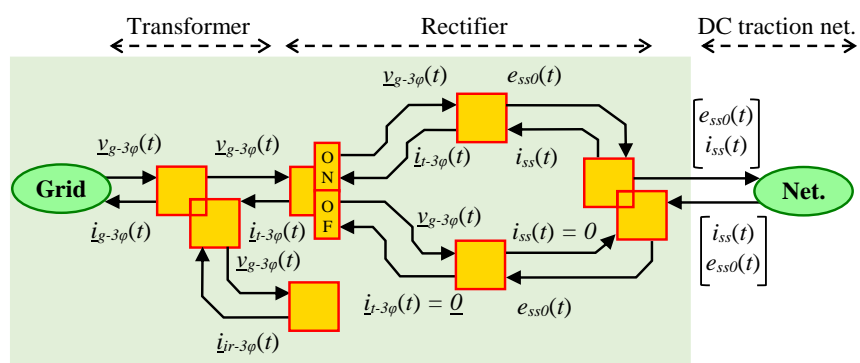

Fig. 6. EMR of the equivalent model of the TPS. 


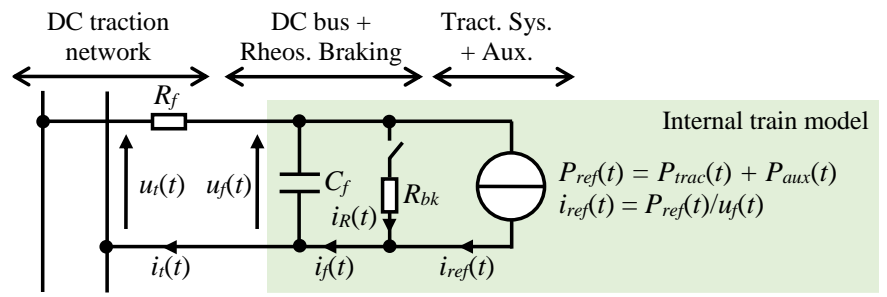

Fig. 7. Equivalent electric circuit of the vehicle.

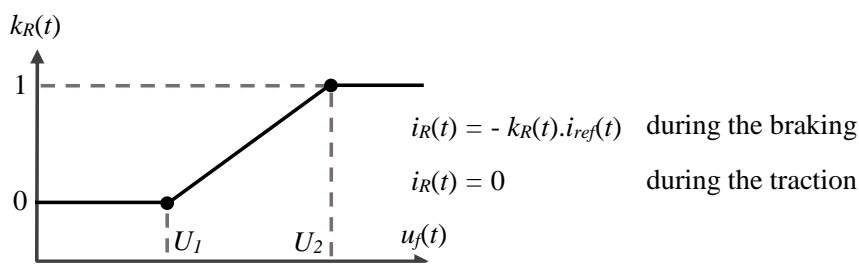

Fig. 8. Squeeze control characteristics (rheostatic braking).

The input filter model and the squeeze control have huge impacts on the simulation approach and the solving procedure of the entire railway system. It will be discussed in Section IV.

\section{Model of the DC Traction Network}

The model of the DC traction network assumes linear resistance distribution along the line. The resistance of each line segment $R_{n e t}(t)$ represents the resistance of the third rail (or catenary) plus the return rail (or ground) between two subsystems (TPS or vehicle). It is a position-dependent parameter that varies with the movement of the trains.

\section{SPECIFIC DC RAILWAY SimUlation APPROACHES}

Three specific approaches for the simulation of complete DC railway systems are described. An example is considered with 1 TPS and 2 trains to illustrate the different approaches (Fig. 9). The 3 approaches use the well-known Modified Nodal Analysis (MNA) to solve the DC traction network [15], [16]. The MNA is generally expressed by (1), where $G$ is the conductance matrix of the DC network (with $G_{\text {net-ik }}=R_{\text {net-ik }}{ }^{-1}$ ), $\underline{R}$ is the matrix that consider the different input resistances (i.e. $R_{s s-i}$ or $R_{f-i}$ ) of the voltage source subsystems, $\underline{B}$ is a matrix corresponding to the Kirchhoff currents equations and is composed of 1 for the voltage source nodes whose branch relations are introduced. The vectors $J$ and $E$ include respectively the variables of known initial currents and voltages based on the outputs of the train and TPS models (current or voltage sources). Then, the vectors $\underline{V}$ and $I$ are the unknown variables, respectively the nodes voltages and the currents of the voltage sources subsystems. These unknown variables are obtained by inverting equation (1) and are used to define the reaction variables of the different models of the subsystems, which are simulated on the traction network.

Note that the TPS model is the same for the 3 different approaches. In on-state, it corresponds to a voltage source, which imposes the voltage $\mathrm{e}_{s s}(t)$. The unknown variable $i_{s s}(t)$ is obtained from $\underline{I}$ (MNA results). In off-state, it corresponds to a current source, which imposes a current $J_{i}=0$. The unknown variable $e_{s s}(t)$ is obtained from $\underline{V}$. One can note that the MNA formulation is strongly dependent on the models of the different subsystems. Indeed, the chosen models define the inputs and outputs of the MNA, and thus the formulation. More explanations will be provided in the final paper.

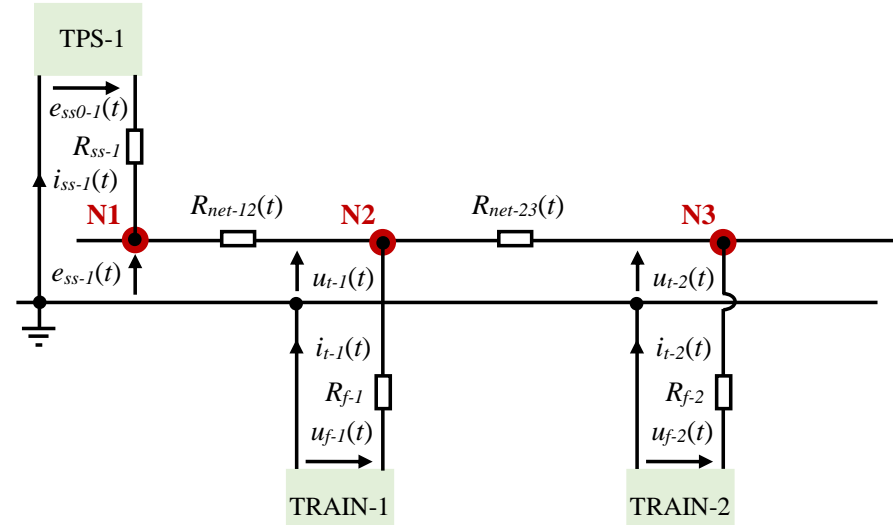

Fig. 9. Example of railway system with 1 TPS and 2 trains.

$$
\left[\begin{array}{ll}
\underline{G} & -\underline{B} \\
B^{t} & -\underline{R}
\end{array}\right]\left[\begin{array}{l}
\frac{V}{I} \\
\underline{G}
\end{array}\right]=\left[\begin{array}{l}
\frac{J}{E} \\
\underline{E}
\end{array}\right], \quad \text { then } \quad\left[\frac{V}{I}\right]=\left[\begin{array}{ll}
\underline{G} & -\underline{B} \\
\underline{B} & -\underline{R}
\end{array}\right]^{-1}\left[\begin{array}{l}
\underline{J} \\
\underline{E}
\end{array}\right]
$$

\section{A. Dynamic model (DYN)}

The first simulation approach is based on a dynamic model of the train input filter (Fig. 7) (Fig. 11.a). The DC bus imposes the voltage $u_{f}(t)$ as inputs for the DC network and the brake, traction, and auxiliary subsystems. The vehicle is thus a voltage source $m$ connected to a node $i$. The internal DC bus current $i_{f}(t)$ is calculated by the train model according to the currents $i_{\text {ref }}(t)$ (Fig. 7) and $i_{R}(t)$ (imposed by the squeeze control in Fig. 8). Furthermore, the DC bus model imposes to the MNA the voltage $u_{f}(t)$. The corresponding unknown variable $i_{t}(t)$ (current exchanged between the train and the DC network) is obtained from the MNA results with the element $I_{m}$ (vector $I$ ). More explanations and equations will be provided in the final paper.

\section{B. Quasi-Static model 1 (QS1)}

The QS1 model can be considered as representative of the conventional approach used in most of the existing simulation tools [5], [9]. This model neglects the DC bus and considers the train as a pure current source $i_{t}(t)$ (Fig. 11.b), with $i_{t}(t)$ equal to $i_{f}(t)$. Furthermore, $i_{R}(t)$ and $i_{\text {ref }}(t)$ are calculated from the voltage value $u_{f}(t)$ of the previous simulation step-time. The current $i_{t}(t)$ is thus imposed as a known variable in the MNA vector $\underline{J}$. The corresponding unknown $u_{t}(t)$ is obtained from the MNA results $(\underline{V})$. The actual DC bus voltages $u_{f}(t)$ is then estimated with (2).

$$
u_{f}(t)=u_{t}(t)-R_{f} i_{t}(t)
$$

One drawback of this approach, contrary to the dynamic model-based approach, is the inability to deal with the case where all the TPSs are blocked. In such case, there is no voltage reference in the DC network, which is disconnected from the AC grid. It is therefore assumed that no energy is exchanged within the entire system: the voltages all over the DC network are imposed to the rated value and the currents are imposed to zero. More details will be provided in the final paper.

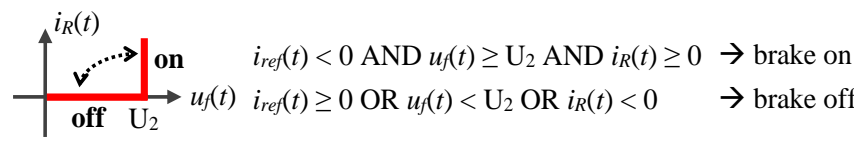

Fig. 10. Squeeze control characteristics (rheostatic braking) 

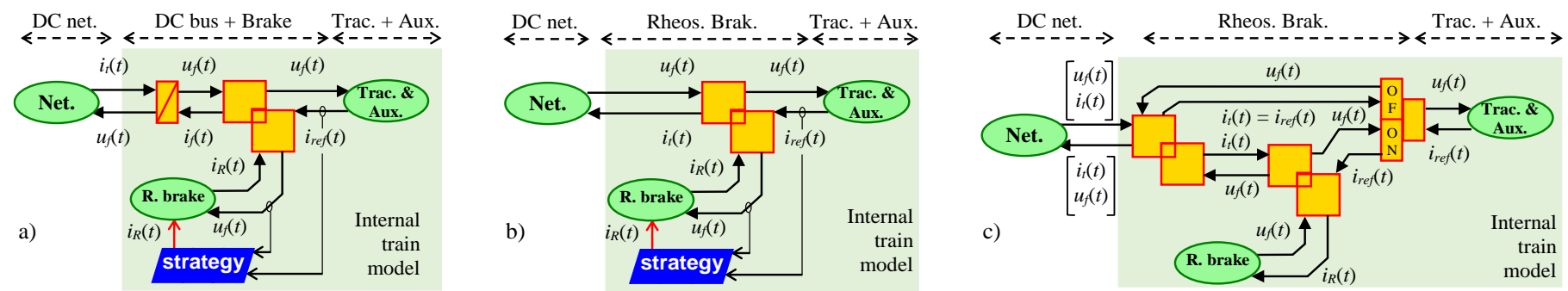

Fig. 11. EMR of the vehicle: a) Dynamic model, b) Quasi-static model 1, c) Quasi-static model 2.

\section{Quasi-Static model 2 (QS2)}

The QS2 model is an intermediary approach that considers current-voltage switching sources for the train model (Fig. 11.c). The current $i_{R}(t)$ is null $\left(i_{t}(t)=i_{\text {ref }}(t)\right)$ if the brake is not activated (off). In such case, the train is a current source and imposes $i_{t}(t)$ to the MNA. The unknown variable $u_{f}(t)$ is obtained from the MNA results and (2). If the brake is activated (on), the train is considered as a voltage source $m$, which regulates the voltage to its maximal value $U_{2}$ and imposes $u_{f}(t)=U_{2}$ to the MNA. The unknown variable $i_{t}(t)$ is obtained from $I_{m}$ (vector $\underline{I}$ ) and the current $i_{R}(t)$ is calculated with (3).

$$
i_{R}(t)=i_{t}(t)-i_{\text {ref }}(t)
$$

This approach neglects the DC bus and the squeeze control between $U_{1}$ and $U_{2}$ (Fig. 8). Other switching conditions are thus used to activate the brake (Fig. 10) (current/voltage sources). More details will be provided in the final paper.

\section{ANALYSIS OF THE SiMULATION RESUlTS}

The results obtained by the 3 approaches are compared and analyzed according to the case study (section II) with the reference power $P_{\text {ref }}(t)=P_{\text {trac }}(t)+P_{\text {aux }}(t)$ (Fig. 12.a). The results in terms of energy in the TPSs are quite similar. The authors therefore focus in this paper on the source of errors, which is concentrated when all TPSs are blocked. In this situation, the DC traction system is completely isolated from the $\mathrm{AC}$ grid and there is no bus that actuates as slack. However, energy exchanges are still possible within the DC network. First, the periods when all TPSs are blocked are identified and are then referred to as "blocked periods". The powers provided by all TPSs with the dynamic model are presented (Fig. 12.b). The black signal is added to highlight these periods. All methods present a similar number of instants (between 9 and $10 \%$ of the simulation time) in which all TPSs are blocked at the same time.
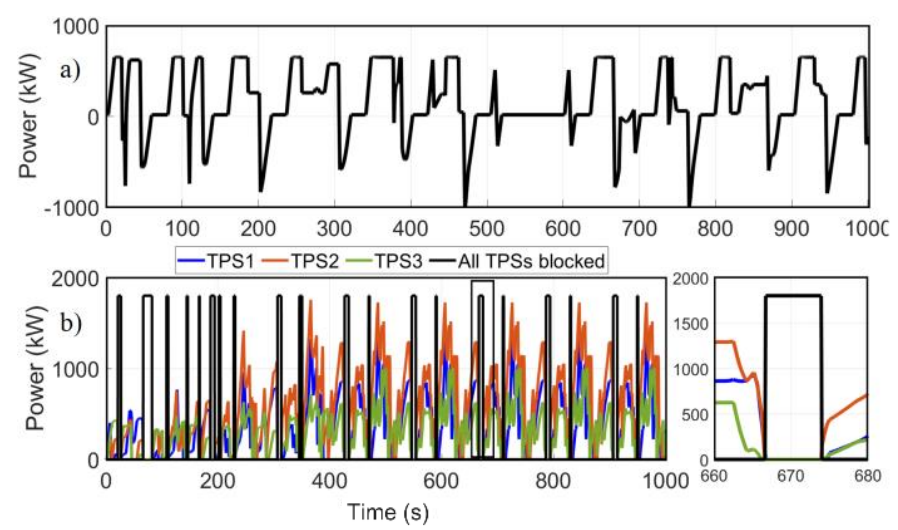

Fig. 12. a) Train reference power $\left(P_{\text {ref }}(t)=P_{\text {trac }}(t)+P_{\text {aux }}(t)\right)$, b) TPSs powers.
The third rail or catenary voltage $u_{t}(t)$ and the current $i_{t}(t)$ of Train 6 are analyzed to highlight the differences between the 3 approaches (Fig. 13). A zoom is provided for a blocked period. The DYN model (blue) gives accurate estimations even during the blocked periods. DYN has been experimentally validated in [12] and is considered as reference in this paper. QS1 is not able to estimate voltages and power flows during the blocked periods (10\% of the time) because it returns the trivial solution (no currents on the line, and DC voltages equal to the rated value). QS2 results (black) give better results than QS1 because it can estimate the energy recovery and the voltages values during the blocked periods. However, there is still an error in comparison to the DYN simulation due to the squeeze control simplification.

An energetic study quantifies the impacts of each approach on the estimation of the internal power flows during the blocked periods. It can be noted that these power flows have no impact on the energy consumption on the AC grid because the railway system is disconnected from the grid during the blocked periods. Nevertheless, it has an impact on the DC network behavior and its different subsystems. Table I summarizes for each method the total reference energy $E_{\text {ref }}\left(\right.$ from $\left.P_{\text {ref }}(\mathrm{t})\right)$ and actual energy $E_{c a t}$ injected or absorbed from the catenary by all the trains. The positive (absorbed energy $E(+)$ ) and negative (recovered energy $E(-))$ are separated to have clear analysis on the requested energy and the regeneration capacity of all trains. The reference energy is the same for the 3 approaches. The difference between $E_{r e f}$ and $E_{c a t}$ corresponds to the losses in the filters and the brakes. The errors on the absorbed and recovered energies with QS1 (vs DYN) are important during the blocked periods (100\%) because the solver returns only the trivial solution. The differences are also not negligible during the entire simulation, with errors of $4 \%$ and $14.8 \%$, respectively on the absorbed and recovered energy by all trains. The energetic error for QS2 is negligible on the entire simulation and is $4 \%$ during the blocked periods.

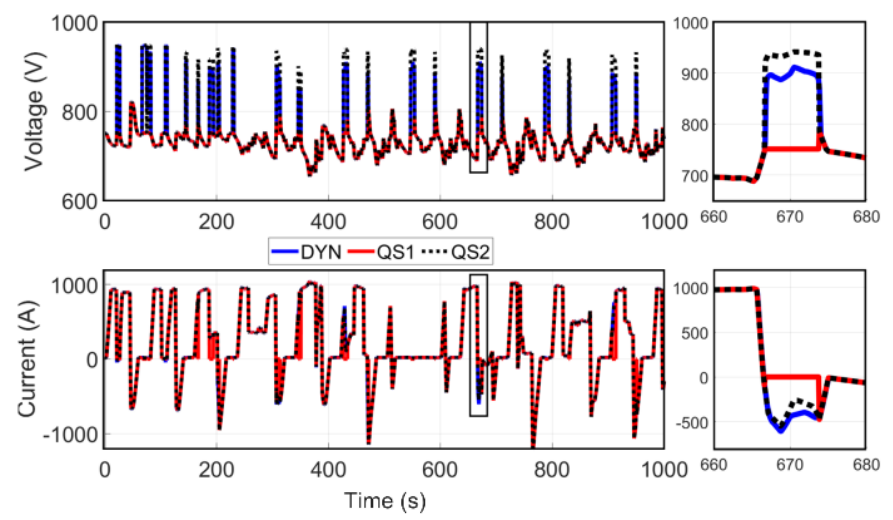

Fig. 13. Third rail or catenary voltage and current of Train 6 with the 3 models. 
TABLE I. ENERGETIC STUDY OF POWER FLOWS WITHIN THE DC TRACTION NETWORK

\begin{tabular}{|c|c|c|c|c|c|c|c|c|c|c|c|c|}
\hline & \multicolumn{2}{|c|}{ REF (kWh) } & \multicolumn{2}{|c|}{ DYN (kWh) } & \multicolumn{2}{|c|}{ QS1 (kWh) } & \multicolumn{2}{|c|}{ QS2 (kWh) } & \multicolumn{2}{|c|}{ QS1 vs DYN $(\%)$} & \multicolumn{2}{|c|}{ QS2 vs DYN (\%) } \\
\hline & $\mathrm{E}_{\mathrm{ref}}(+)$ & $\mathrm{E}_{\text {ref }}(-)$ & $\mathrm{E}_{\text {cat }}(+)$ & $\mathrm{E}_{\text {cat }}(-)$ & $\mathrm{E}_{\mathrm{cat}}(+)$ & $\mathrm{E}_{\mathrm{cat}}(-)$ & $\mathrm{E}_{\text {cat }}(-)$ & $\mathrm{E}_{\mathrm{cat}}(-)$ & $\mathrm{E}_{\mathrm{cat}}(+)$ & $\mathrm{E}_{\mathrm{cat}}(-)$ & $\mathrm{E}_{\text {cat }}(+)$ & $\mathrm{E}_{\text {cat }}(-)$ \\
\hline Entire simulation (1000s) & 452.5 & -142.9 & 469.7 & -130.8 & 450.8 & -111.4 & 469.6 & -130.7 & 4.02 & 14.83 & 0.02 & 0.07 \\
\hline All TPSs blocked periods & 19.0 & -28.9 & 19.4 & -19.8 & 0.0 & -0.0 & 18.6 & -18.9 & 100.00 & 100.00 & 4.12 & 4.54 \\
\hline
\end{tabular}
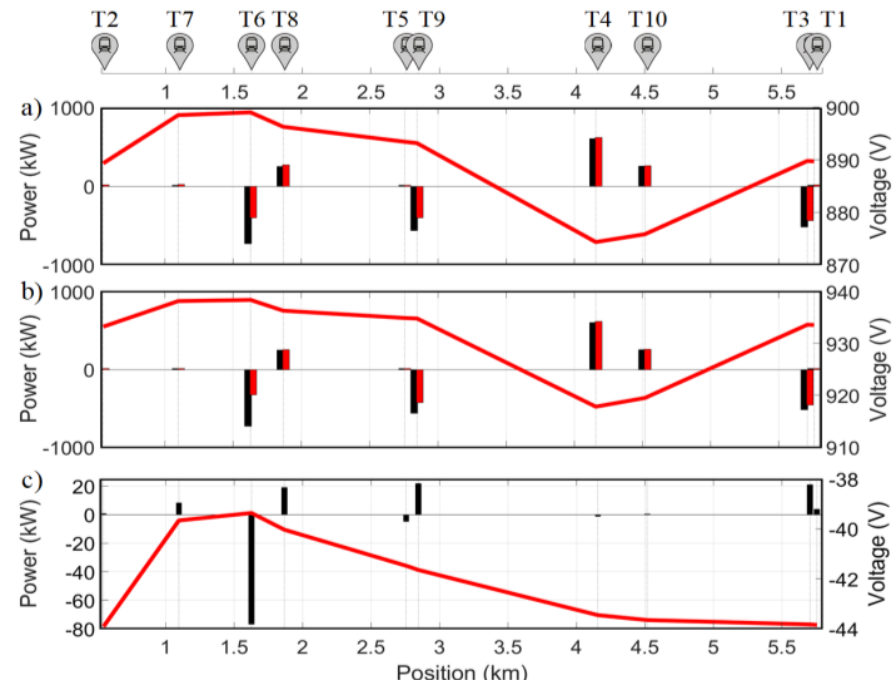

Fig. 14. Simulation results at the instant 670s: a) DYN results, b) QS2 results, c) differences between DYN and QS2.

Fig. 14 presents a specific instant (670s) where all TPSs are blocked (QS1 is not presented). The horizontal axis corresponds to the positions. The vertical axis represents the voltages at the junction with the catenary (right scale - solid red line) and the reference power $P_{\text {ref }}(t)$ and actual power exchanged with the DC network (left scale - black and red bars respectively). The sum of all power references of all trains in that specific instant is $-637 \mathrm{~kW}$. There is a power surplus that leads to increasing the voltages and blocking all TPSs. The net power injection at this specific instant is $23.4 \mathrm{~kW}$ with DYN and $16.1 \mathrm{~kW}$ with QS2 (0kW with QS1). Regarding the voltage profile, QS2 provides a higher voltage profile due to the squeeze control simplification, which explains the lower power injection with this method. The accuracy of the estimation of the DC voltages and the regeneration capacity of the trains is crucial to perform a study assessment of innovative subsystems. Indeed, these new subsystems are particularly used when the line receptivity is low. In addition, their controls are often based on the voltage value. It is thus essential to develop accurate and fast simulation tools. More analysis will be provided in the final paper.

\section{CONCLUSION}

The paper presents and compares 3 different methodologies for the simulation of DC railway systems. The study is focused on the periods in which all the traction power substations are blocked at the same time since the results in other conditions are quite similar. A first simulation approach, based on an experimentally validated dynamic model (DYN), is presented and used as reference for comparisons. Quasi-static method 1 (QS1) provides the trivial solution and leads to significant errors compared to DYN. Quasi-static method 2 (QS2) provides better results than QS1 but needs to be further improved to obtain more accurate solutions closer to the DYN method. Future research will thus focus on improving the accuracy of the QS2 method to obtain equivalent results to those obtained with the DYN method. In addition, the potential reduction of the computation time with this new method will be studied.

\section{REFERENCES}

[1] R. Barrero, J. van. Mierlo, and X. Tackoen, "Energy savings in public transport," IEEE Veh. Technol. Mag., vol. 3, no. 3, pp. 26-36, Sep. 2008.

[2] T. R. Eastham, "Running off the rails," IEEE Spectr., vol. 40, no. 1, pp. 66-69, jan. 2003.

[3] M. L. Pastor, L. G.-T. Rodriguez, and C. V. Vélez, "Flywheels store to save: Improving railway efficiency with energy storage," IEEE Electrification Mag., vol. 1, no. 2, pp. 13-20, Dec. 2013.

[4] W. Wang, M. Cheng, Y. Wang, B. Zhang, Y. Zhu, S. Ding, and W. Chen, "A novel energy management strategy of onboard supercapacitor for subway applications with permanent-magnet traction system," IEEE Trans. Veh. Technol., vol. 63, no. 6, pp. 2578-2588, Jul. 2014.

[5] A.-M. Gee, and R.-W. Dunn, "Analysis of trackside flywheel energy storage in light rail system," IEEE Trans. Veh. Technol., vol. 64, no. 9, pp. 3858-3869, Oct. 2015.

[6] Y.-S. Tzeng, R.-N. Wu, and N. Chen, "Electric network solutions of DC transit systems with inverting substations," IEEE Trans. Veh. Technol., vol. 47, no. 4, pp. 1405-1412, Nov. 1998.

[7] A. Rufer, D. Hotellier, and P. Barrade, "A supercapacitor-based energy storage substation for voltage compensation in weak transportation network," IEEE trans. Power Del., vol. 19, no. 2, pp. 629-636, Apr. 2004.

[8] Y. Cai, M.-R. Irving, and S.-H. Case, "Iterative techniques for the solution of complex DC-rail-traction systems including regenerative braking," in Proc. of IEE - Generation, Transmission and Distribution, vol. 142, no. 5, pp. 445-452, Sep. 1995.

[9] M.-Z. Chymera, A.-C. Renfrew, M. Barnes, and J. Holden, "Modeling electrified transit systems," IEEE Trans. Veh. Technol., vol. 59, no. 6, pp. 2748-2756, Jul. 2010.

[10] L. Abrahamsson, and L. Söder, "Fast estimation of relations between aggregated train power system data and traffic performance," IEEE Trans. Veh. Technol., vol. 60, no. 1, pp. 16-29, Jan. 2011.

[11] P. Arboleya, B. Mohamed, and I. El-Sayed, "DC railway simulation including controllable power electronics and energy storage devices," IEEE Trans. Power Syst., vol. 33, no. 5, pp. 5319-5329, Sep. 2018.

[12] C. Mayet, P. Delarue, A. Bouscayrol, E. Chattot, and J.-N. Verhille, "Comparison of different EMR-based models of Traction Power Substations for energetic studies of subway lines," IEEE Trans. Veh. Technol., vol. 65, no. 3, pp. 1021-1029, Mar. 2016.

[13] C. Mayet, L. Horrein, A. Bouscayrol, P. Delarue, J.-N. Verhille, E. Chattot, and B. Lemaire-Semail, "Comparison of different models and simulation approaches for the energetic study of a subway," IEEE Trans. Veh. Technol., vol. 63, no. 2, pp. 556-565, Feb. 2014.

[14] A. Bouscayrol, J.-P. Hautier, and B. Lemaire-Semail, "Systemic design methodologies for electrical energy systems - Chapter 3: Graphic formalism for the control of multi-physical energetic systems: COG and EMR," ISTE Ltd and John Wiley \& Sons, Inc., 2012.

[15] C.-W. Ho, A. Ruehli, and P. Brennan, "The modified nodal approach to network analysis," IEEE Trans. Circuits Syst., vol. 22, no. 6, pp. 504 509, Jun. 1975.

[16] V. Acary, O. Bonnefon, and B. Brogliato, "Nonsmooth modelling and simulation of switched circuits," Springer Science \& Business Media, 2010.

[17] More references will be provided in the final paper. 